\title{
An image dehazing algorithm based on double priors constraint
}

\author{
Kun Liu, Shiping Wang, Linyuan $\mathrm{He}^{*}$, Duyan $\mathrm{Bi}$, and Shan Gao \\ Air Force Engineering University, Xi'an 710038, China
}

\begin{abstract}
Aiming at the color distortion of the restored image in the sky region, we propose an image dehazing algorithm based on double priors constraint. Firstly, we divided the haze image into sky and non-sky regions. Then the Color-lines prior and dark channel prior are used for estimating the transmission of sky and non-sky regions respectively. After introducing color-lines prior to correct sky regions restored by the dark channel prior, we get an accurate transmission. Finally, the local media mean value and standard deviation are used to refine the transmission to obtain the dehazing image. Experimental results show that the algorithm has obvious advantages in the recovery of the sky area.
\end{abstract}

\section{Introduction}

Image dehazing methods based on the degradation model have drawn a great attention in recent years. After analyzing lots of outdoor haze-free image, dark channel prior was put forward [1] to estimate the initial transmission. This method can achieve satisfactory recovery effects in most natural scenes, which has been widely used for image dehazing at present. However, due to the fact that the dark channel prior does not apply to the sky region, there is a halo in the sky region of the image after dehazing. At the same time, the color of the image is distorted. Therefore, some improved algorithms have been proposed. Tarel use median filtering to estimate the transmission [2], which reduces the complexity of the algorithm. Zhu proposed an image dehazing algorithm based on the color attenuation prior [3], which improved the running speed of the algorithm. However, the algorithm relies too much on the color information and cannot handle the entire fog image well.

In general, the above algorithm has a poor effect on the restoration of the sky region, and it is likely to cause color distortion. As a result, some improved algorithms for the sky region have been proposed successively. Li [4] separately estimated the transmission of the non-sky region and the sky region, suppressing the color distortion effectively, but the color of restored image is dark. Sui [5] used Markov random fields to estimate the transmission. Although there is no color distortion in the sky area, this method does not handle well in the details of the distant images.

In order to achieve the dehazing in the sky region, this paper proposes an image dehazing algorithm based on double priors constraint. After dividing the sky region, the proposed method introduce color-lines prior to correct sky regions restored by the dark

\footnotetext{
*Corresponding author: hal1983@163.com
} 
channel prior, then we obtain the accurate transmission through the fusion of two transmission. Finally we obtain haze-free images by the atmospheric degradation model.

\section{Background}

\subsection{Atmospheric Degradation Model And The Dark Channel Prior}

The scattering effect of light leads to the production of haze image. The causes of haze image degradation is shown in figure 1.

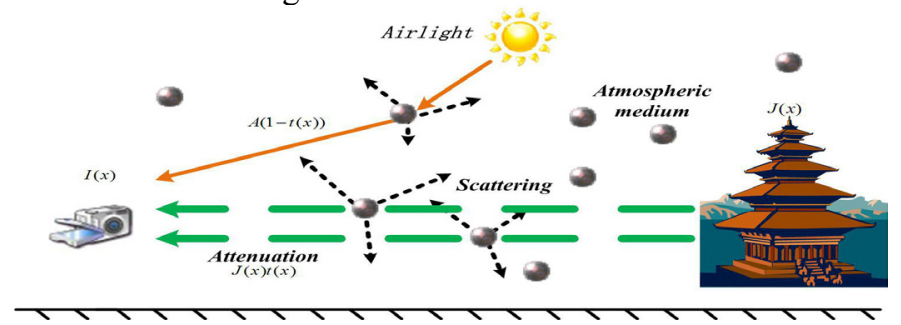

Fig 1. Formation of a hazy image.

where $\boldsymbol{I}(\boldsymbol{x})$ denotes the degraded image, $\boldsymbol{J}(\boldsymbol{x})$ is the haze-free image to be restored, A is the global atmospheric light intensity, which is assumed to be a constant in this paper, $\boldsymbol{t}(\boldsymbol{x})$ is the transmission, reflecting the degree of attenuation of the scene. Then the atmospheric degradation model is proposed by McCartney [6], which can be written as equation (1):

$$
I(x)=J(x) t(x)+A(1-t(x))
$$

\subsection{The dark channel prior}

In most of the local patch except the sky region, the intensity of some pixels in at least one color channel is very small, which is called the dark channel prior. For a fog-free image $\boldsymbol{J}$ $(x)$. There are:

$$
J^{\mathrm{dark}}(x)=\min _{y \in \Omega(x)}\left(\min _{c \in\{r, g, b\}}\left(J^{\mathbf{c}}(x)\right)\right)=0
$$

where $\boldsymbol{J}^{\boldsymbol{c}}(\boldsymbol{x})$ represents the R,G,B color channels of image $\boldsymbol{J}(\boldsymbol{x})$, and $\boldsymbol{\Omega}(\boldsymbol{x})$ denotes the local patch centered on $\boldsymbol{x}$. Due to the large intensity of the pixels in the sky region, there is no dark color point with an intensity close to zero. While performing image defogging, the difference between the color values of the three channels will be amplified, resulting in color distortion of the image.

\subsection{The Color-lines Prior}

Beased on a statistical analysis of a large number of natural images. Omer found that the pixels in the local area obey one-dimensional distribution in the $R G B$ color space, showing a line, which is called Color-lines. Then, Fattal[7] relies on this discovery to define a model in a local image region. He[1] estimated the transmission based on the distance from the origin to the color line. By which we can estimate an accurate transmission. According to the Color-Lines prior and surface reflection properties, the recovered haze-free image can be represented as equation (3):

$$
J(x)=l(x) \bar{R}, \quad x \in \Omega
$$

where $\bar{R}$ is a vector in the $R G B$ color space, which represents the relative luminance of the reflected light in each color channel. The scalar $\boldsymbol{l}(\boldsymbol{x})$ is the radiation at the pixel $\mathrm{x}$ in the 
local patch $\boldsymbol{\Omega}$. In other words, $\bar{R}$ can be regarded as the reflectivity of the surface, while $\boldsymbol{l}(\boldsymbol{x})$ is the surface shadow. Since the transmission $\boldsymbol{t}(\boldsymbol{x})$ is smoothed locally[8], we regard it as a constant. So in a small local patch, equation (1) can be rewritten as:

$$
I(x)=t l(x) \bar{R}+(1-t) A=l(x) R+(1-t) A, x \in \Omega
$$

where $R=t \bar{R}$. In addition, Based on the above analysis, it is known that the pixels $x(x \in \Omega)$ following this model differ only in surface shadow. According to equation (4), we can know that the $\boldsymbol{I}(\boldsymbol{x})$ corresponding to these pixels presents a one-dimensional distribution in the $R G B$ color space. The color-line corresponding to these local areas changes with $\boldsymbol{l}(\boldsymbol{x})$. As indicated by the blue dotted line in figure 2, its direction is the same as the reflectivity $\boldsymbol{R}$, which is denoted by the purple line. Under the effect of atmospheric light, the color-line does not exceed the origin. To which it is $\boldsymbol{A}(\mathbf{1 - t})$ from the origin. So the intersection of the atmospheric light and the color line indicates the distance from the atmospheric light direction to the color line.
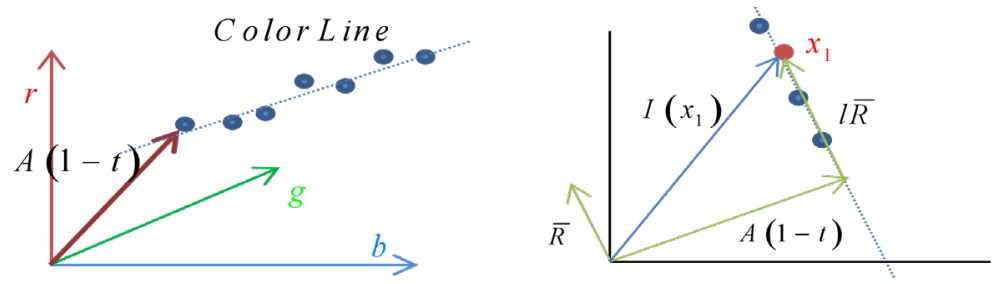

Fig. 2. Color-lines.

Figure 3 shows the distribution of Color-lines in different regions of the image. We can learn from the figure that this model is apply to sky region. For the purpose of correcting the transmission obtained by the dark channel prior, we can estimate the transmission of sky region through the Color-lines prior.

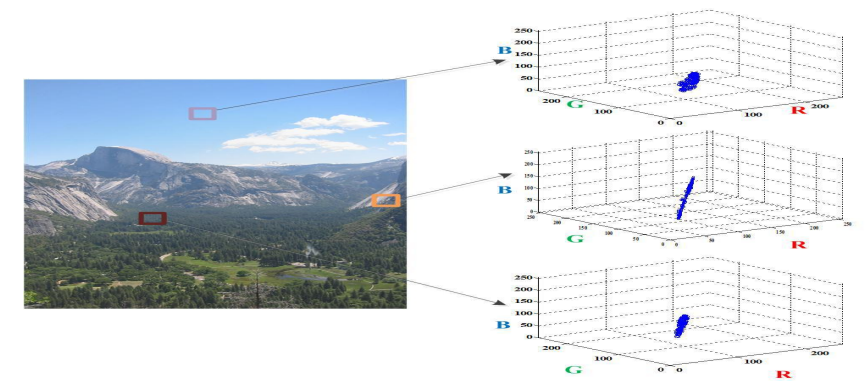

Fig. 3. Color-lines of different patches.

\section{The framework of our algorithm}

\subsection{Sky Region Segmentation}

In most haze images containing sky regions, there are clear differences between sky and non-sky regions. According to which, we can achieve the separation of sky and non-sky regions.

As a result of the noise interference, it is difficult to achieve accurate segmentation for an image with noise interference at the junction directly. Therefore, the proposed method achieves the division of the sky region by quadratic segmentation. At first, we converted natural images to grayscale images. Next,we segmented the sky region from the image according to equation (5) to obtain a preliminary segmentation result: 


$$
\tilde{t_{1}}(x)= \begin{cases}I_{\text {gray }}(x) \geq 255, & x \in s k y \\ 0 \leq I_{\text {gray }}(x)<255, & x \notin s k y\end{cases}
$$

Here $\boldsymbol{I}_{\text {gary }}$ is gray intensity of the pixel $\boldsymbol{x}$. The difficulty in segmenting the sky region lies in the determination of the segmentation threshold $\boldsymbol{T}$. Weather it is correct or not is crucial to the segmentation result. In order to ensure the correct segmentation of the sky region, we use the Otsu Algorithm to achieve the second segmentation, where the threshold $\mathrm{T}$ is obtained by equation (5):

$$
T=\arg \max _{0 \leq i \leq N-1}\left\{\sigma^{2}(t)\right\}
$$

The relationship between $\sigma^{2}$ and $\boldsymbol{t}$ is expressed as follows:

$$
\begin{aligned}
\sigma^{2} & =w_{\text {sky }}\left(\mu_{\text {sky }}-\mu_{r}\right)^{2}+w_{\text {nonsky }}\left(u_{\text {nonsky }}-u_{r}\right)^{2} \\
& =w_{\text {sky }} w_{\text {nonsky }}\left(\mu_{\text {sky }}-u_{\text {nonsky }}\right)
\end{aligned}
$$

where $\boldsymbol{\mu}_{\boldsymbol{r}}$ refers to the gray average of the image, and $\boldsymbol{W}_{\text {sky }}$ and $\boldsymbol{W}_{\text {non-sky }}$ are the probability distributions of the sky and non-sky regions. Based on the above quadratic segmentation method, the sky region and the non-sky region can be distinguished accurately. The segmentation result is shown in figure 4 :
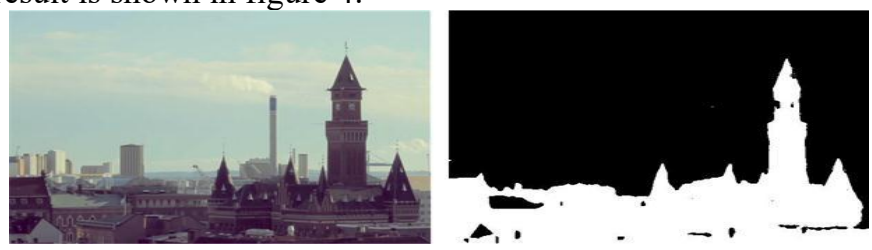

Fig. 4. Color-lines of different patches.

\subsection{The Estimation of Initial Transmission}

After the segmentation, we make use of the Color-lines prior and the dark channel prior for the sky and non-sky regions to obtain the initial transmission respectively. For a color-line that has been obtained in the segmented sky region, we can obtain the transmission by counting its distance from the origin according to figure 2. As known from the second section, that the deviation distance can be regarded as the intersection of the color-line and

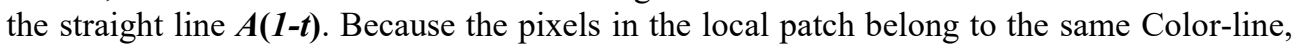
we randomly select two pixels $\boldsymbol{x}_{1}$ and $\boldsymbol{x}_{2}$ in the local patch $\boldsymbol{\Omega}$, whose intensity are $\boldsymbol{I}\left(\boldsymbol{x}_{1}\right)$ and $\boldsymbol{I}\left(\boldsymbol{x}_{\boldsymbol{1}}\right)$ respectively. Therefore, the color-line can be expressed as:

$$
\left(\frac{I\left(x_{2}\right)-I\left(x_{1}\right)}{x_{2}-x_{1}}\right) x+I\left(x_{1}\right), x_{1}, x_{2} \in \Omega
$$

Here, $\frac{I\left(x_{2}\right)-I\left(x_{1}\right)}{x_{2}-x_{1}}$ denotes the direction of the Color-line. As shown in figure 2, it is in the same direction as $\boldsymbol{R}$. Furthermore, the pixel intensity is only different in $\boldsymbol{l}(\boldsymbol{x})$ in the local patch, so equation (8) can be written as :

$$
l(x) R+I\left(x_{1}\right), x_{1}, x_{2} \in \Omega
$$

We can estimate $\mathrm{t}$ according to equation (10). Followed by which, a rough estimation of the transmission $\tilde{t}_{s k y}(x)$ in sky region is obtained by equation (7).

$$
\min \left\|l(x) R+I\left(x_{1}\right)-s A\right\|, \quad s=1-t
$$

On the other hand, for non-sky regions, the initial transmission $\tilde{t}_{\text {nonsky }}(x)$ can be obtained by the dark channel prior. In general, the haze image has different transmission between the sky area and the non-sky area. To avoid the halo phenomenon after dehazing, 
we fuse the transmission $\tilde{t}_{n o n s k y}(x)$ and $\tilde{t}_{\text {sky }}(x)$ according to equation (11) to obtain a rough transmission $\tilde{t}(x)$ of the entire image.

$$
\tilde{t}(x)=w(x) \tilde{t}^{\prime}{ }_{\text {nonsky }}(x)+(1-w(x)) \tilde{t}_{\text {sky }}(x)
$$

Here $\boldsymbol{w}(\boldsymbol{x})$ is a weighted function for fusion, expressed as:

$$
w(x)=\frac{1}{M N} \sum_{i=1}^{M} \sum_{j=1}^{N}\left[I_{\text {gray }}(x)-u\right]^{2}, x \in \Omega
$$

where $\boldsymbol{M N}$ represents the number of pixels, $\boldsymbol{I}_{\text {gray }}(\boldsymbol{x})$ represents the gray intensity of the pixels, and $u$ is the mean value of the local window $\boldsymbol{\Omega}$. When it comes to the sky region or the bright region, the smaller the difference between the pixels is, the greater the weight of $\tilde{t}_{s k y}(x)$ is than the $\bar{t}_{n o n s k y}(x)$. So that the correction of the transmission in the sky region can be achieved.

\subsection{The Correction of The Transmission}

Because the Color-lines prior do not apply to all local patches in the image. Therefore, in order to obtain accurate transmission, we need to modify the rough transmission $\tilde{t}(x)$.which is essential for dehazing results. Since the ideal transmission should be changing smoothly except for the edge of image, the transmission $\tilde{t}(x)$ can be optimized by equation (11) to improve its accuracy.

$$
\begin{gathered}
\sum_{x} \frac{[\tilde{t}(x)-\hat{t}(x)]^{2}}{\sigma^{2}(x)}+\lambda \sum_{x} \sum_{y \in N_{x}} \frac{[\tilde{t}(x)-\tilde{t}(y)]^{2}}{\|I(x)-I(y)\|^{2}} \\
\hat{t}(x)=\max \left\{t(x), 1-\min _{c \in\{R, G, B\}}\left\{\frac{I^{c}(x)}{A}\right\}\right\}
\end{gathered}
$$

where $\lambda$ denotes a regularization coefficient, $N_{x}$ denotes the nearest four pixels around the pixel point $\boldsymbol{C}^{x}, \boldsymbol{\mu}(\boldsymbol{x})$ is the mean valve and $\boldsymbol{\sigma}(\boldsymbol{x})$ is the standard deviation of $\tilde{t}(x)$. which reflects the degree of dispersion of the data set. The first term in equation (13) is a fidelity term that minimizes the difference between $\tilde{t}(x)$ and $\hat{t}(x)$, allowing the transmission to be estimated only within the range that satisfies the color-lines prior. The second item is a penalty item that indicates the spatial relationship of the image. Through the local difference, the recovered transmission can retain the detailed information in the original image better.

\section{Experimental results}

In order to verify the effectiveness of the proposed method, we compares experimental results with the current mainstream dehazing algorithm to analyze the applicability of this algorithm. At the same time, we compared with the results of the restoration of the composite image to comprehensively demonstrate the effect of our algorithm. Finally, we introduce objective indicators to achieve the qualitative analysis of experimental results.

\subsection{Analysis of Experimental Results}

The comparison of the proposed method with other methods is shown in figure 5. It can be summed up that He's method can achieve effective dehazing, However, its results in the sky regions is unsatisfactory. As we can see, color distortions appear in the sky regions of several images. Though Fattal'method retains more detailed information in the flat region, 
it has poor ability of margin preservation and the restored image is darker. Meng'method has clear details in the image after dehazing, but there is still distortion in the sky region. Compared with these methods, the proposed method is more natural and clear in the details of the image. At the same time, it corrects the color distortion in the sky region, and greatly improves the image quality.

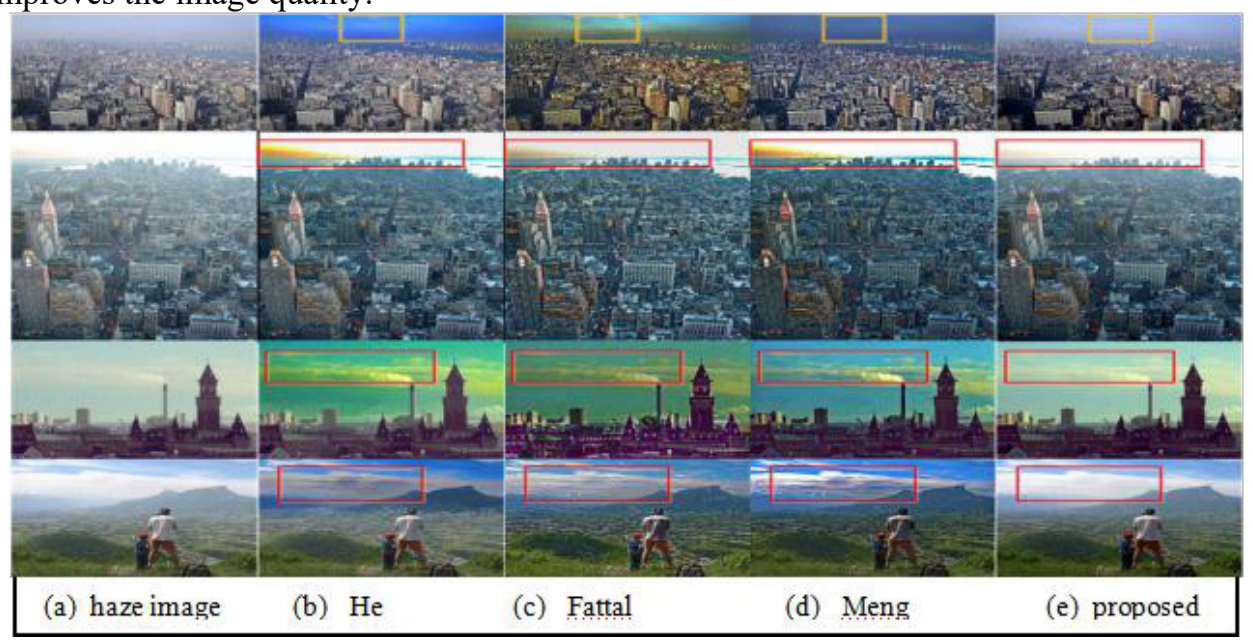

Fig. 5. Dehazing results in sky region.

In order to further verify the effectiveness of the proposed, we analyzes the results of the synthetic images, which is shown in figure 6.

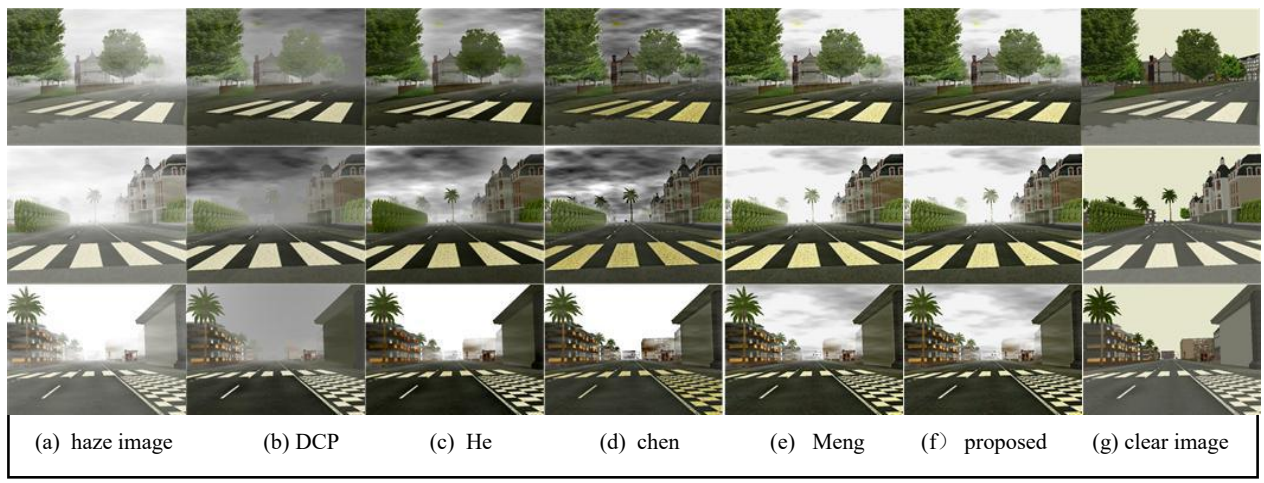

Fig. 6. Dehazing results for synthetic images.

It can be seen that the proposed algorithm has obvious advantages in the recovery of the sky area. For example, dark cloud patches rarely appear in the sky area, effectively correcting color distortion in the sky area. Other than, the restored image is more natural and conforms to human visual characteristics.

\subsection{Analysis of Quantitative Comparison}

To further quantify the proposed method, we use the image entropy and peak signal-tonoise ratio (PSNR) to analyze the various algorithms in figure 5 quantitatively.

The entropy of the image represents the average information contained in the image. The PSNR is the most direct method in image quality evaluation, which mainly reflects the fidelity of the image and the integrity of the structure. Table 1 is the entropy and PSNR of the results in figure 5 . 
We found that the entropy of the image restored by the proposed method is the largest, indicating that the recovered image contains more information. Because the proposed algorithm improves the defect of the dark channel prior to the sky region and the recovered image is more clear and natural, so the PSNR is the largest. In general, the proposed method maintains more detailed information than other methods.

Table 1. Entropy/PSNR of experimental results.

\begin{tabular}{|c|c|c|c|c|c|}
\hline Image & $\begin{array}{c}\text { Haze } \\
\text { Image }\end{array}$ & He & Fattal & Meng & Proposed \\
\hline $\mathbf{( 1 )}$ & 7.4588 & $7.5018 / 16.8165$ & $7.4267 / 13.2542$ & $7.5174 / 13.5746$ & $7.6933 / 19.4782$ \\
\hline $\mathbf{( 2 )}$ & 7.1267 & $7.1413 / 18.4563$ & $6.8725 / 16.3861$ & $7.2354 / 18.3673$ & $7.4365 / 18.5481$ \\
\hline $\mathbf{( 3 )}$ & 7.0518 & $7.1621 / 5.6349$ & $7.1318 / 14.4772$ & $7.3568 / 16.4821$ & $7.4309 / 22.7654$ \\
\hline $\mathbf{( 4 )}$ & 7.3556 & $7.6589 / 13.8653$ & $7.5624 / 13.5746$ & $7.5698 / 14.2589$ & $7.8670 / 19.3689$ \\
\hline
\end{tabular}

\section{Conclusion}

In this paper, we proposes an image dehazing algorithm based on double priors constraint. The proposed method has a great effect on the dehazing in the sky region. While achieving color retention, the restored image retains more image detail. What's more, the image quality is improved significantly. However, we can't get ideal dehazing image when dealing with denser images. This is a major research direction for follow-up work.

\section{Acknowledgments}

This work is supported by the National Natural Science Foundation of China, under Grants 61701524.

\section{References}

1. He KaiMing, Sun Jian, Tang Xiaoou et al. Single image haze removal using dark channel prior [C].Processdings of the IEEE Conference on Computer Visionand Pattern recognition Miami: IEEE, 2009: 1956-1963.

2. Tarel J P, Hautiere N. Fast visibility restoration from a single color or gray level image [C]. Proceedings of the 12th IEEE International Conference on Computer Vision Kyoto. IEEE, 2009: 2201-2208.

3. Zhu Qingsong, Mai Jiaming, Shao Ling. A Fast Single Image Haze Removal Algorithm Using Color Attenuation Prior[J].IEEE Transactions on image Processing, 2015: 3522-3532.

4. Li J Y, Hu Q W, Ai M Y, Yan J. Image haze removal based on sky region detection and dark channel prior [J]. Journal of Image and Graphics, 2015, 20(4):0514-0519

5. Sui Ping, BI Duyan, HE Linyuan, MA Shiping Higher-order Markov Random fields defogging based on Color Lines[J].Journal of Electronics \& Information Technology, 2016, 38(9): 2405-2409

6. McCartney E J. Optics of Atmosphere: Scattering by Molecules and Particles [M]. New York: John Wiley and Sons, 1976: 23-32.

7. R Fattal. Dehazing using color-lines[J]. ACM Transactions on Graphics, , 34(2014)

8. Meng G., Wang Y., Duan J, Xiang S, Pan, C. Efficient image dehazing with boundary constraint and contextual regularization. In: ICCV. pp. 617-624 (2013). 